\title{
DIFERENCIAS Y SIMILITUDES ENTRE LAS ESTRATEGIAS DE GESTIÓN INTEGRADA PARA LA PREVENCIÓN Y CONTROL DEL DENGUE EN COLOMBIA Y PERÚ
}

\author{
Raimundo Castro-Orozco ${ }^{1, a}$; Nelson Alvis-Guzmán¹,b; Rubén Gómez-Arias²,c
}

\begin{abstract}
RESUMEN
Se analizaron y compararon aspectos esenciales de las Estrategias de Gestión Integrada (EGI) Nacional Colombia y Nacional Perú, mediante una revisión narrativa de las fuentes disponibles, con la finalidad de identificar semejanzas y diferencias en dos países andinos en el contexto del dengue. Se identificaron diferencias relacionadas con la valoración del problema, la conformación de grupos de participantes y la información cuantitativa aportada por los indicadores de cumplimiento. Las debilidades identificadas en la EGI-Dengue Nacional Colombia 2006-2010 son consideradas en una nueva versión de la estrategia (2012-2021). Se documentó el impacto epidemiológico de la implementación de la EGI Nacional Colombia 2006-2010. Adicionalmente, se observó un incremento gradual en la tasa de incidencia reportada que pudiera estar relacionado con el fortalecimiento del sistema de vigilancia integral de la EGI Nacional Perú.
\end{abstract}

Palabras clave: Dengue; Políticas públicas de salud; /prevención \& control (fuente: DeCS BIREME).

\section{DIFFERENCES AND SIMILARITIES IN APPROACH OF INTEGRATED STRATEGY FOR DENGUE PREVENTION AND CONTROL BETWEEN COLOMBIA AND PERU}

\begin{abstract}
We analyzed and compared two Integrated Management Strategies for Dengue Prevention and Control (IMS-dengue Colombia and IMS-dengue Peru), through a narrative review of available literature, in order to identify common and dissimilar patterns in two Andean countries with epidemiological differences in the context of dengue disease. We were able to identify differences related to: formal assessment of problem, formation of groups of actors, and quantitative information provided by performance indicators. These limitations identified in IMS-dengue Colombia 2006-2010 were overcome in a new version of the strategy (IMS-dengue Colombia 2012-2021). We were able to document an epidemiological impact of implementation of IMS-dengue Colombia 2006-2010. Additionally, a gradual increase was observed in incidence rates of dengue cases that could be related to the strengthening of surveillance system of IMSdengue Peru.
\end{abstract}

Key words: Dengue; Public health policy; /prevention \& control (source: MeSH NLM).

\section{INTRODUCCIÓN}

Entre las fiebres hemorrágicas virales transmitidas por artrópodos, el dengue es la más frecuente en el mundo, afecta a todos los continentes $y$, recientemente, con reportes de nuevos casos en áreas no endémicas, como Estados Unidos y Europa. En consecuencia, el dengue se constituye como un problema de salud pública vigente y sentido a nivel global ${ }^{(1,2)}$.
A principio de los años 70 , las campañas de control vectorial lograron erradicar al Aedes (Stegomyia) aegypti, en 21 países del continente americano. Sin embargo, la falta de sostenimiento o el abandono de los programas de control terminaron en la reinfestación de estos países. En la actualidad, se observa una tendencia creciente de la incidencia del dengue en estos países, con picos epidémicos cada vez mayores ${ }^{(2,3)}$.

\footnotetext{
Universidad de San Buenaventura. Cartagena de Indias, Colombia

Universidad de Antioquia. Medellín, Colombia

Químico farmacéutico, magíster en Microbiología; ${ }^{\mathrm{b}}$ médico, doctor en Economía y Gestión de la Salud; ${ }^{\mathrm{c}}$ médico, doctor en Salud Pública Recibido: 18-05-15 Aprobado: 02-09-15
}

Citar como: Castro-Orozco R, Alvis-Guzmán N, Gómez-Arias R. Diferencias y similitudes entre las estrategias de gestión integrada para la prevención y control del dengue en Colombia y Perú. Rev Peru Med Exp Salud Publica. 2015;32(4):801-7. 
Ante el evidente fracaso de las intervenciones basadas en abordajes lineales como el control de la reproducción del vector por rociado con insecticidas de acción residual, para el año 2001, la Organización Panamericana de la Salud (OPS), y la Organización Mundial de la Salud (OMS), formularon nuevos lineamientos dirigidos a promover programas de prevención y control del dengue basados en cuatro pilares: participación comunitaria, educación sanitaria, voluntad política y coordinación intrasectorial ${ }^{(4)}$.

A pesar de su diversidad, la región de América Latina comparte patrones geográficos, bióticos, económicos y culturales que trascienden las fronteras y la exponen a enfermedades transmisibles por vectores. Para lograr avances significativos en la prevención y control de estas enfermedades en los países de la región, es necesario integrar políticas públicas sostenibles a través del desarrollo de programas intersectoriales, el fortalecimiento de la legislación sanitaria y la participación activa de las comunidades susceptibles, tal como lo conciben las Estrategias de Gestión Integrada (EGI) Dengue ${ }^{(4,5)}$. Para los años 2006 y 2007 se dio inició a la implementación de esta estrategia en Colombia y Perú, respectivamente ${ }^{(6)}$.

Se reconocen diferencias en el comportamiento epidemiológico del dengue en estos dos países de la subregión andina, tal cual lo muestran los reportes de casos para el año 2014, donde la mayor letalidad por dengue reportada en esta subregión se presentó en Perú, pero la incidencia de dengue en Colombia fue, aproximadamente, nueve veces mayor a la reportada en Perú para este mismo año ${ }^{(7,8)}$. Lo anteriormente descrito pudiera ser el resultado de debilidades y dificultades particulares que pudieran estar afectando la efectividad de la implementación de la EGI-Dengue en estos dos países andinos.

En el presente artículo se realizó una revisión narrativa de la literatura disponible relacionada con la implementación de políticas públicas en salud para la prevención y control del dengue en Colombia y en Perú, que permitiera identificar situaciones o patrones comunes y disímiles en dos países andinos con diferencias epidemiológicas en el contexto del dengue.

\section{METODOLOGÍA DE LA REVISIÓN}

Para localizar los documentos relacionados con la EGIDengue se realizó una búsqueda bibliográfica en los sitios web de la OPS, del Ministerio de Salud y Protección Social-MSPS de Colombia y del Ministerio de SaludMINSA del Perú. También se realizó una búsqueda en Internet en el buscador "Google Académico" utilizando los siguientes descriptores: "EGI-Dengue, estrategias regionales, estrategias nacionales, Colombia y Perú".

Tras la combinación de los descriptores utilizados se obtuvieron 28 fuentes de información. Se seleccionaron aquellos documentos que informasen sobre aspectos esenciales de la planificación e implementación de la estrategia; tres de ellas fueron de especial utilidad para el estudio: EGI Nacional Colombia 2006-2010 (9), EGI Nacional Colombia 2012-2021 (10) y EGI Nacional Perú 2007-2011 (11). Estos documentos fueron sometidos a un análisis formal de acuerdo con el modelo descrito por Walt et al. ${ }^{(12)}$, para el análisis de una política en salud.

EGI NACIONAL COLOMBIA 2006-2010 VS. EGI NACIONAL PERÚ 2007-2011

Se realizó un análisis formal de los documentos, procurando identificar los elementos esenciales de la política en ambos países, tales como: objeto de la política, actores responsables involucrados, propósitos, indicadores, fuentes de verificación, supuestos y resultados a obtener por componente de la estrategia (Tabla 1).

Objeto de la política. Se revisaron los documentos buscando precisar la manera cómo se formuló el problema en cada país. Para el caso de la EGI Nacional Colombia, no se hizo referencia explícita al problema en términos ambientales, vectoriales, económicos o patrones culturales de riesgo. En cambio, parece ser más completa la concepción del problema en la EGI Nacional Perú, aunque su valoración omite la descripción del impacto de la enfermedad en términos económicos.

Análisis de los involucrados. En la EGI Nacional Perú se destaca la colaboración de un centro de reconocimiento internacional en el estudio de enfermedades infecciosas y tropicales, universidades, organizaciones socialescomunales y de estamentos gubernamentales con funciones en salud ambiental y de desarrollo de recursos humanos. En contraste, no se registra ninguna de estas participaciones en el documento específico de EGI Nacional Colombia, aunque en la elaboración de la guía de vigilancia entomológica se reporta la participación técnica de universidades, centros de investigación, organizaciones no gubernamentales e instituciones gubernamentales ${ }^{(13)}$.

Análisis de los indicadores, propósitos, fuentes de verificación y supuestos/riesgos. En la $E G I$ Nacional Colombia, los indicadores de cumplimiento se caracterizan por presentar poca información cuantitativa, lo cual podría ser una limitación en el caso de que se quiera realizar una evaluación de la estrategia al comparar la meta planeada y el desempeño logrado. 
Tabla 1. Comparación de elementos esenciales entre la EGI Nacional Colombia 2006-2010 y la EGI Nacional Perú 2007-2011

\begin{tabular}{|c|c|c|}
\hline Elementos & EGI Nacional Colombia 2006-2010 & EGI Nacional Perú 2007-2011 \\
\hline $\begin{array}{l}\text { Objeto de } \\
\text { la política }\end{array}$ & $\begin{array}{l}\text { Valoración del problema: } \\
\text { - Comparación de la frecuencia nacional de } \\
\text { casos de dengue y dengue hemorrágico con } \\
\text { la frecuencia de casos reportados en la región } \\
\text { andina o en el continente americano }\end{array}$ & $\begin{array}{l}\text { Valoración del problema: } \\
\text { - Descripción del comportamiento de los reportes de caso } \\
\text { (2001-2007) } \\
\text { - } \text { Reportes de brotes de dengue } \\
\text { - } \quad \text { Impacto del comportamiento estacional sobre la intensidad de } \\
\text { estos brotes } \\
\text { - Poblaciones o ciudades más afectadas } \\
\text { - Densidad del vector y su relación con la variación estacional } \\
\text { - Descripción de factores de riesgo asociados y conductas o } \\
\text { variables condicionantes de la transmisión del dengue } \\
\text { - Representación gráfica del comportamiento de los casos } \\
\text { reportados de dengue en Perú,1990-2007 }\end{array}$ \\
\hline $\begin{array}{l}\text { Análisis de } \\
\text { los involu- } \\
\text { crados }\end{array}$ & $\begin{array}{ll}\text { - } & \text { OPS/OMS } \\
\text { - } & \text { Asesores internacionales OPS/OMS (Panamá, } \\
& \text { Brasil, Cuba, EI Salvador y Honduras) } \\
\text { - } & \text { MSPS (dependencias relacionadas a ETV y, } \\
& \text { salud pública) } \\
\text { - } & \text { INS } \\
\text { - } & \text { Coordinadores Departamentales de ETV } \\
\text { - } & \text { Departamentos Administrativo de Salud } \\
\text { - } & \text { Cuatro Secretarias Departamentales de Salud }\end{array}$ & $\begin{array}{ll}\text { - } & \text { OPS/OMS } \\
\text { - } & \text { USAID } \\
\text { - } & \text { UNICEF } \\
\text { - } & \text { Gobierno Regional y Local } \\
\text { - } & \text { MINSA (dependencias relacionadas a salud ambiental, } \\
& \text { epidemiología, promoción de salud, comunicaciones, etc.) } \\
\text { - } & \text { INS } \\
\text { - } & \text { Comités consultores } \\
\text { - } & \text { ONG } \\
\text { - } & \text { Universidades } \\
\text { - Instituto de Medicina Tropical Alexander van Humboldt } \\
\text { - } \text { Organizaciones sociales y comunales }\end{array}$ \\
\hline
\end{tabular}

\begin{tabular}{|c|c|c|}
\hline Propósitos & $\begin{array}{l}\text { Contribuir a la reducción de la morbilidad, } \\
\text { mortalidad y la carga socioeconómica causada } \\
\text { por dengue en la población colombiana en riesgo. }\end{array}$ & $\begin{array}{l}\text { Contribuir a reducir en forma sostenida el impacto social y } \\
\text { económico de dengue en la población peruana entre los años } \\
2007 \text { a } 2011\end{array}$ \\
\hline Indicadores & $\begin{array}{l}\text { - Porcentaje en la reducción de la incidencia } \\
\text { por dengue (2206-2010), monitoreado } \\
\text { semanalmente y evaluado anualmente } \\
\text { - Tasa de mortalidad por dengue evaluada } \\
\text { anualmente } \\
\text { - Porcentaje en la reducción de la letalidad por } \\
\text { dengue hemorrágico (2006-2010), evaluado } \\
\text { anualmente } \\
\text { - Recursos y compromisos procedentes de } \\
\text { sectores públicos, privados y de organizaciones } \\
\text { comunitarias incorporados activamente a la } \\
\text { estrategia }\end{array}$ & $\begin{array}{l}\text { - Mantenimiento de la letalidad por dengue hemorrágico por } \\
\text { debajo de } 2 \% \\
\text { - Detección y control temprano de todos los brotes y epidemias } \\
\text { - Reducción en el número de brotes de dengue } \\
\text { - Porcentaje de vinculación y compromiso del sector privado (50 } \\
\% \text { ), sector público }(70 \%) \text { y de organizaciones relacionadas a la } \\
\text { EGl-Dengue }(80 \%)\end{array}$ \\
\hline $\begin{array}{l}\text { Fuentes de } \\
\text { verificación }\end{array}$ & $\begin{array}{l}\text { - } \text { Actos administrativos } \\
\text { - Planes intersectoriales } \\
\text { - } \text { Sistema de información en salud pública }\end{array}$ & $\begin{array}{ll}\text { - } & \text { Acuerdos } \\
\text { - } & \text { Convenios } \\
\text { - } & \text { Actas de compromisos (instituciones públicas y privadas) } \\
\text { - } & \text { Informes técnicos de cumplimiento de acuerdos y compromisos } \\
\text { - } & \text { Informes entomológicos y epidemiológicos } \\
\text { - } & \text { Informes del sistema de vigilancia epidemiológica }\end{array}$ \\
\hline Supuestos & $\begin{array}{l}\text { - Mantenimiento de la estabilidad política y } \\
\text { socioeconómica }\end{array}$ & $\begin{array}{l}\text { - Mantenimiento de la estabilidad política y socioeconómica } \\
\text { - Ocurrencia de cambios climáticos o desastres naturales } \\
\text { significativos }\end{array}$ \\
\hline Resultados & $\begin{array}{l}\text { - Fortalecimiento de la red de entomología y de } \\
\text { la red nacional de laboratorio } \\
\text { - Generación de cambios conductuales } \\
\text { (aplicación de metodología COMBI) } \\
\text { - Estratificación del riesgo de transmisión del } \\
\text { dengue en Colombia } \\
\text { - Mejoras en la detección, diagnóstico, } \\
\text { tratamiento y notificación oportuna de los } \\
\text { pacientes con dengue }\end{array}$ & $\begin{array}{l}\text { - Generación de un sistema de vigilancia en salud pública } \\
\text { integrado a la red nacional de laboratorio } \\
\text { - Reducción en la mortalidad asociada } \\
\text { - Control o disminución de los criaderos del vector } \\
\text { - Modificación favorable de los comportamientos de riesgo frente } \\
\text { al vector, a nivel individual, de familia y comunitario } \\
\text { - Participación activa de los sectores públicos y privados en la } \\
\text { formulación y ejecución de políticas en salud }\end{array}$ \\
\hline
\end{tabular}

OPS: Organización Panamericana de la Salud; OMS: Organización Mundial de la Salud; MSPS: Ministerio de Salud y Protección Social; INS: Instituto Nacional de Salud; ETV: enfermedades transmitidas por vectores; USAID: Agencia de los Estados Unidos para el Desarrollo Internacional; UNICEF: Fondo de las Naciones Unidas para la Infancia; ONG: Organizaciones No Gubernamentales; EGI Nacional Colombia 2006-2010 (9); EGI Nacional Perú 2007-2011 (11). 
Es interesante señalar que el mismo documento en cuestión incluye indicadores mejor elaborados, en cuanto a la información cuantitativa se refiere, tales como: disminución del $50 \%$ de la tasa de incidencia de dengue en Colombia en un período de cinco años, mantener la tasa de letalidad por dengue hemorrágico por debajo del $1 \%$ anualmente en Colombia, en un período de cinco años y, al menos, el $70 \%$ de los sectores públicos y privados de Colombia incorporados activamente a la EGI-Dengue. Estos tres indicadores corresponden a una meta similar, pero para un período de cumplimiento diferente (2005-2010).

Sin bien es cierto que los propósitos de la estrategia en los dos países son similares, se observan marcadas diferencias en cuanto a los indicadores correspondientes a la EGI Nacional Perú, ya que se proyectó un porcentaje menor en la vinculación y compromiso del sector privado a la EGI-Dengue, y un mantenimiento de la letalidad por dengue hemorrágico por debajo del $2 \%$. Adicional a esto, se planificó la detección y control temprano de todos los brotes y epidemias, acompañado con la reducción en el número de brotes.

Resultados esperados de la EGI por componentes. A pesar de que la EGI Nacional Colombia formuló el problema exclusivamente en términos epidemiológicos, la matriz consolidada por componentes incluye diferentes actividades para conseguir otros resultados como: fortalecimiento de la red de entomología y de la red nacional de laboratorios, generación de cambios conductuales como resultado de la aplicación de la metodología COMBI, estratificación del riesgo de transmisión del dengue en Colombia y mejoras en la detección, diagnóstico, tratamiento y notificación oportuna de los pacientes con dengue.

En ambos países, los resultados de la estrategia aluden al cambio de los patrones culturales de riesgo pero sin diferenciar entre el entorno urbano o rural.

\section{IMPLANTACIÓN DE LA EGI NACIONAL COLOMBIA 2012-2021}

En un documento borrador emitido por el MSPS se proyecta la EGI-Dengue para un nuevo decenio. Adicional a los participantes involucrados en la elaboración de la EGI Nacional Colombia 2006-2010, en este documento borrador la participación se amplía a cinco universidades públicas y privadas, Servicio Nacional de Aprendizaje-SENA, diez secretarías departamentales o seccionales de salud, una empresa social del Estado-ESE, dos instituciones prestadoras de salud-IPS y la colaboración de más de 80 docentes, investigadores o personal vinculado con la vigilancia epidemiológica.
En esta nueva proyección de la EGI, se observa una definición mucho más clara y apropiada del problema, ya que no valora el problema solo en términos de la comparación de reportes de casos, sino que explica su tendencia ascendente a partir de una conjunción de factores asociados al virus, al vector y a las poblaciones expuestas. También, se describen los patrones de endemicidad regional y nacional, acompañando su fundamentación con información relacionada al comportamiento de las tasas de incidencia de dengue y dengue grave.

Sumado a esto, presenta la tendencia del dengue en el país (1990-2010), la tendencia de letalidad por dengue (1998-2010) y el consolidado de casos de dengue grave y letalidad en Colombia (1998-2010). En la descripción del problema se citan dos investigaciones: un estudio de costo y un análisis del reporte de casos y de la tasa de letalidad hasta la semana epidemiológica 50 del 2011.

Con todo lo anterior, se puede observar que la nueva proyección de la EGI en Colombia, busca fundamentar la dinámica del problema en el uso y valoración de evidencias, aunque todavía no ha considerado la determinación de los contextos económicos y culturales sobre la transmisión del dengue.

La evaluación externa, realizada en el año 2008, permitió identificar fortalezas y dificultades en cada uno de los componentes de la estrategia. Entre las dificultades se destacaron: falta de recurso humano competente, capaz y con liderazgo en la gerencia del programa, ausencia de un análisis integrado de información, poca participación de entidades nacionales, regionales y locales asociadas a salud en la toma de decisiones, poca efectividad de los canales de comunicación entre la comunidad educativa y las entidades de salud, entre otras.

A pesar de tratarse de un borrador provisional, sus novedades permiten visualizar un avance significativo, tanto en la planificación como en la implementación de la EGI-Dengue que debería derivar, en los próximos diez años, en una mejor forma de prevenir y controlar la transmisión del dengue en el territorio colombiano.

En los cuatro años anteriores a la implementación de la EGI Nacional Colombia, el promedio de la tasa de incidencia fue de 236,3 casos de dengue por 100 mil habitantes. En los primeros cuatros años de implementación de la estrategia se observó una marcada disminución en la tasa de incidencia nacional del dengue (185,5 casos de dengue por 100 mil habitantes); sin embargo, en 2010, se reportó la más alta tasa de incidencia en el país (657 casos de dengue por 100 mil habitantes) ${ }^{(14)}$.

Esta epidemia nacional ha sido asociada con varios factores, tales como: la presencia del fenómeno El Niño, la adaptación del vector a climas más templados y a la 
presencia del vector tanto en el entorno urbano como en el rural ${ }^{(15)}$. El hecho demuestra que la estrategia EGl-Dengue fue incapaz de prevenir la epidemia; pero también es plausible asumir que si esta misma conjunción de factores se hubiera dado en ausencia de la implementación de la estrategia, el escenario epidémico pudo haber sido de mayor magnitud que el reportado ${ }^{(14)}$.

Aunque no se encontró ninguna referencia que describiera la prolongación de la EGI-Dengue en Colombia durante el año 2011, la tasa de incidencia de dengue disminuyó en comparación con el año anterior ${ }^{(16)}$. Este comportamiento decreciente pudiera ser coherente con la idea planteada en el hipotético escenario descrito anteriormente.

Ahora bien, durante los primeros tres años de la implementación de la EGI Nacional Colombia 20122021, la tasa de incidencia del dengue ha mostrado un comportamiento ascendente ${ }^{(7,17,18)}$. Es más, se puede observar el nivel de epidemia nacional alcanzado en el año 2013 (Figura 1), a pesar de que esta situación epidemiológica se caracterizó por una baja notificación de los casos compatibles con dengue ${ }^{(19)}$. Lo anterior ha llevado a algunos autores a considerar que la epidemia nacional de dengue de este año pudo tener una magnitud aún mayor.

\section{IMPLANTACIÓN DE LA EGI NACIONAL EN PERÚ 2007-2011}

Con respecto a la implementación de la EGI Nacional Perú, se observa un cambio en la tendencia de la tasa de incidencia del dengue de los tres años previos (2004-2006) en comparación con los cinco años de implementación de la estrategia (Figura 1) ${ }^{(22-26)}$. Este incremento gradual pudiera estar relacionado con el fortalecimiento del sistema de vigilancia integral derivado de la implementación de la estrategia. La circulación del genotipo asiático/americano del DENV-2 en el Perú explica el comportamiento epidémico del dengue en el último año de la EGI Nacional Perú (27).

Con la metodología de búsqueda de información aplicada en este estudio no se pudo encontrar ninguna otra versión de la EGI Nacional Perú, sin embargo, en la página web del MINSA, para el 2011, se reportan logros derivados de la implementación de la estrategia ${ }^{(28)}$. Además, existe un reporte realizado por el MINSA de Perú sobre ciertas experiencias exitosas en la prevención y control del dengue en el contexto de la EGI-Dengue, a través de la implementación de la metodología de comunicación para impactar en conductas-COMBI ${ }^{29)}$, coherentes con el objetivo del Plan Nacional Multisectorial e Intergubernamental de Prevención y Control en el Perú (30).

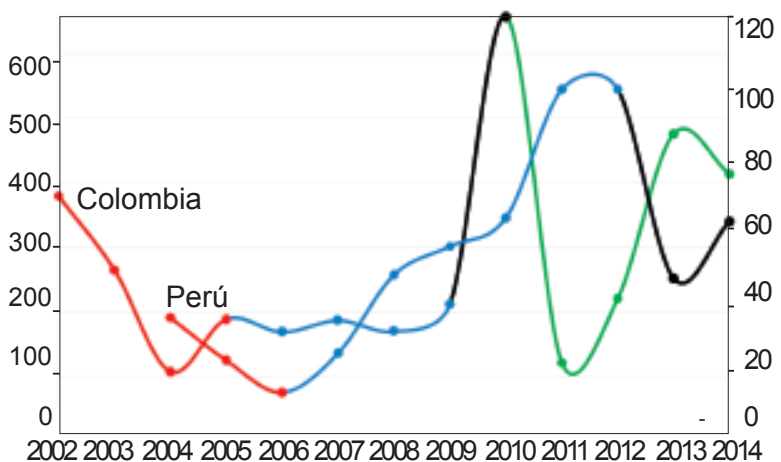

Figura 1. Tendencias de las tasas de incidencia ajustada de dengue (por 100 mil habitantes) en Colombia y Perú, en diferentes períodos de tiempo de acuerdo con la implementación de EGI (Colombia, rojo: 2002-2005; azul: 2006-2010; negro: 2011 y verde: 2012-2014) ${ }^{(7,14,16-18)}$ (Perú, rojo: 2004-2006; azul: 2007-2011 y negro: 2012-2014) (20-26,34)

Igualmente, se pudieron identificar diferentes experiencias o documentos relacionados con la implementación de los componentes de la EGI-Dengue, tales como la "Guía clínica para la atención de casos de dengue en el Perú" (31), la "Norma Técnica de Salud para la Implementación de la Vigilancia y Control Vectorial en Perú" (32) y el "Plan integrado de vigilancia, prevención y control de dengue" (33).

Por otra parte, los tres años siguientes al período de implementación de la EGI Nacional Perú muestran una tendencia decreciente en la tasa de incidencia pero manteniendo un ligero incremento en comparación con la tasa de incidencia promedio para el período comprendido entre 2007 y 2011 (69 casos por 100 mil habitantes vs. 57,8 casos por 100 mil habitantes, respectivamente) ${ }^{(22-26,34)}$.

Es de anotar que este estudio puede presentar las limitaciones que se han descrito para toda revisión narrativa ${ }^{(35)}$, pero los resultados soportan la necesidad de fortalecer la integración regional de los países, en pro de la búsqueda de posibilidades de cooperación que nos permitan fortalecer las políticas públicas en salud y la capacidad técnica del recurso humano, para así poder hacer frente a los desafíos venideros del dengue en América.

\section{CONCLUSIONES}

A pesar de su diversidad, la región de América Latina comparte patrones geográficos, bióticos, económicos y culturales que trascienden las fronteras y la exponen a enfermedades transmisibles por vectores. Para lograr avances significativos en la prevención y control de estas enfermedades en los países de la región, es necesario integrar políticas públicas sostenibles a través del desarrollo de programas intersectoriales, 
el fortalecimiento de la legislación sanitaria y la participación activa de las comunidades susceptibles, tal como lo concibe la EGI-Dengue.

Como resultado de la comparación de la elaboración e implementación de esta estrategia en dos países andinos, Colombia y Perú, se identificaron diferencias importantes en cuanto a: la valoración del problema, la conformación de los grupos de participantes involucrados en la elaboración de la estrategia, la información cuantitativa aportada por los indicadores de cumplimiento y el registro de los cambios climáticos que pudieran afectar la sustentabilidad de los beneficios generados por la estrategia.

Cabe destacar que muchas de estas debilidades identificadas en la EGI Nacional Colombia 2006-2010 son superadas en la versión borrador de la estrategia para implementar en el período 2012-2021.

Durante el período de implementación de estas estrategias, se observan en ambos países, incrementos en la notificación de casos de dengue y en las tasas de letalidad asociada, lo cual podría ser consecuencia tanto de ciertas debilidades en la implementación de la estrategia, que aún permanecen sin resolución y que parecen ser más sentidas durante los períodos de epidemias, como del impacto reconocido que tienen ciertas características del hospedador y de los serotipos circulantes del virus del dengue sobre el comportamiento epidemiológico de esta arbovirosis en las poblaciones humanas.

Contribuciones de autoría: RCO, NAG y RGA participaron en la concepción y diseño del artículo, recolección y obtención de resultados, análisis e interpretación de los datos y redacción del artículo. NAG realizo el análisis e interpretación de resultados y revisión crítica del artículo, además RGA participo con el aporte de material de estudio, todos aprobaron la versión final.

Fuentes de financiamiento: autofinanciado

Conflictos de interés: los autores declaran no tener conflictos de interés.

\section{REFERENCIAS BIBLIOGRÁFICAS}

1. Mangold KA, Reynolds SL. A review of dengue fever: a resurging tropical disease. Pediatr Emerg Care. 2013 May;29(5):6659; quiz 670-1. doi: 10.1097/ PEC.0b013e31828ed30e.

2. Meltzer E. Arboviruses and viral hemorrhagic fevers (VHF). Infect Dis Clin North Am. 2012 Jun;26(2):47996. doi: 10.1016/j.idc.2012.02.003.

3. Toan NT, Rossi S, Prisco G, Nante N, Viviani S. Dengue epidemiology in selected endemic countries: factors influencing expansion factors as estimates of underreporting. Trop Med Int Health. 2015 Jul;20(7):840-63. doi: $10.1111 / \mathrm{tmi} .12498$.

4. San Martin JL, Brathwaite-Dick O. La estrategia de gestión integrada para la prevención y el control del dengue en la región de las Américas. Rev Panam Salud Publica. 2007;21(1):55-63.

5. Resolución CD44.R9: Dengue. 44. Consejo Directivo de la Organización Panamericana de la Salud, 22 al 26 de septiembre de 2003. Washington DC: OPS/OMS; 2003.

6. Situación del dengue en Subregión Andina [Internet]. Lima: ORASCONHU; 2012 [citado el 12 de febrero 2015]. Disponible en: http://www.orasconhu.org/sites/ default/files/files/prest $\% 20$ OPS $\% 20$
Reunion\%20dengue\%20gye\%20 may2012\%281\%29.pdf

7. Instituto Nacional de Salud de Colombia. Comportamiento de los eventos de vigilancia en salud pública: Enfermedades transmitidas por vectores. Boletín Epidemiológico Semanal [Internet]. 2014;(53):1721 [citado el 15 de febrero 2015]. Disponible en: http://www.ins.gov.co/ boletin-epidemiologico/Boletn\%20 Epidemiolgico/2014\%20Boletin\%20 epidemiologico\%20semana\%2053.pdf

8. Dirección General de Epidemiología Ministerio de Salud de Perú. Resumen de las enfermedades o eventos bajo vigilancia epidemiológica en el Perú, del 28 de Diciembre del 2014 al 03 de Enero de 2015 [Internet]. Bol Epidemiol (Lima) [Internet]. 2014;23(53):1044-8 [citado el 15 de agosto 2015]. Disponible en: http://www.dge.gob.pe/portal/docs/ vigilancia/boletines/2014/53.pdf

9. Ministerio de Salud y Protección Social de Colombia.Estrategia de gestión integrada nacional Colombia-EGI Nacional Colombia [Internet]. Bogotá: MPS; 2010 [citado el 12 de febrero 2015] Disponible en: http://www2. paho.org/hq/dmdocuments/2010/ EGI-NAL-COL.pdf

10. Ministerio de Salud y Protección Social de Colombia. Estrategia de gestión integrada para la promoción y control de las enfermedades transmitidas por vectores en Colombia, 2012-2021 [Internet]. Bogotá: MPS; 2013 [citado el 13 de febrero 2015]. Disponible en: http://www.proyectomalariacolombia. co/files/msps/EGIETVNacionalversion26demarzode2013.pdf

11. Ministerio de Salud del Perú. Estrategia de gestión integrada de prevención y control de dengue en Perú [Internet]. Perú: MINSA; 2007 [citado el 12 de febrero 2015] Disponible en: http://www2. paho.org/hq/dmdocuments/2010/EGINAL-PER.pdf

12. Walt G, Gilson L. Reforming the health sector in developing countries: the central role of policy analysis. Health Policy Plan. 1994 Dec;9(4):353-70.

13. Ministerio de Salud y Protección Social de Colombia. Gestión para la vigilancia entomológica y control de la transmisión de dengue [Internet]. Bogotá: MPS; 2013 [citado el 12 de febrero 2015]. Disponible en: http://www.ins.gov. co/temas-de-interes/Dengue/03\%20 Vigilancia\%20entomo\%20dengue.pdf

14. Padilla JC, Rojas DP, Sáenz-Gómez R. Dengue en Colombia. Epidemiología de la reemergencia a la hiperendemia. 1ra ed. Bogotá: Guías de Impresión Ltda.; 2012.

15. Pérez JE. La epidemia de dengue en Colombia es un problema de salud 
que no se puede soslayar. Biosalud. 2010;9(1):5-6.

16. Bello SL. Informe final del evento dengue, año 2011 [Internet]. Bogotá: Instituto Nacional de Salud; 2011 [citado el 14 de febrero 2015]. Disponible en: http:// www.ins.gov.co/lineas-de-accion/ Subdireccion-Vigilancia/Informe $\% 20$ de\%20Evento\%20Epidemiolgico/ Dengue\%202011.pdf

17. Romero L. Informe final del evento dengue, año 2012 [Internet]. Bogotá: Instituto Nacional de Salud; 2012 [citado el 14 de febrero 2015]. Disponible en: http://ins.gov.co/ lineas-de-accion/SubdireccionVigilancia/Informe $\% 20 \mathrm{de} \% 20$ Evento\%20 Epidemiolgico/ DENGUE\%20\%202012.pdf

18. Mercado M. Informe final del evento dengue, año 2013 [Internet]. Bogotá: Instituto Nacional de Salud; 2013 [citado el 14 de febrero 2015]. Disponible en: http:// www.ins.gov.co/lineas-de-accion/ Subdireccion-Vigilancia/Informe $\% 20$ de\%20Evento\%20Epidemiolgico/ DENGUE\%202013.pdf

19. Romero L, Pacheco O, de la Hoz F, Díaz FA. Evaluación de la notificación del dengue durante una epidemia, Colombia. Rev Saúde Pública. 2014; 48(6):899-905.

20. Dirección General de Epidemiología, Ministerio de Salud de Perú. Situación de las enfermedades bajo vigilancia epidemiológica en el Perú, al 31 de diciembre de 2005. Bol Epidemiol (Lima) [Internet]. 2005;14(52):116 [citado el 27 de febrero 2015]. Disponible en: http://www.dge.gob. pe/boletines/2005/52.pdf

21. Dirección General de Epidemiología, Ministerio de Salud de Perú. Situación de las enfermedades bajo vigilancia epidemiológica en el Perú, al 30 de diciembre de 2006. Bol Epidemiol (Lima) [Internet]. 2006;15(52):798811 [citado el 27 de febrero 2015] Disponible en: http://www.dge.gob. pe/boletines/2006/52.pdf

22. Dirección General de Epidemiología, Ministerio de Salud de Perú. Situación de las enfermedades bajo vigilancia epidemiológica en el Perú, al 30 de diciembre de 2007. Bol Epidemiol (Lima) [Internet]. 2007;16(52):696751 [citado el 27 de febrero 2015]. Disponible en: http://www.dge.gob. pe/boletines/2006/52.pdf
23. Dirección General de Epidemiología, Ministerio de Salud de Perú. Situación de las enfermedades bajo vigilancia epidemiológica en el Perú, al 27 de diciembre de 2008. Bol Epidemiol (Lima) [Internet]. 2008;17(52):93958 [citado el 27 de febrero 2015]. Disponible en: http://www.dge.gob. pe/boletines/2008/52.pdf

24. Dirección General de Epidemiología, Ministerio de Salud de Perú. Situación de las enfermedades bajo vigilancia epidemiológica en el Perú, al 2 de enero de 2010. Bol Epidemiol (Lima) [Internet]. 2009;18(52):977-1000 [citado el 27 de febrero 2015]. Disponible en: http://www.dge.gob. pe/boletines/2009/52.pdf

25. Dirección General de Epidemiología, Ministerio de Salud de Perú. Situación de las enfermedades bajo vigilancia epidemiológica en el Perú, al 1 de enero de 2011. Bol Epidemiol (Lima) [Internet]. 2010;19(52):1095128 [citado el 27 de febrero 2015]. Disponible en: http://www.dge.gob. pe/boletines/2010/52.pdf

26. Dirección General de Epidemiología, Ministerio de Salud de Perú. Situación de las enfermedades bajo vigilancia epidemiológica en el Perú, al 29 de enero de 2012. Bol Epidemiol (Lima) [Internet]. 2012;21(52):875-907 [citado el 27 de febrero 2015]. Disponible en: http://www.dge.gob. pe/boletines/2012/52.pdf

27. Dirección General de Epidemiología, Ministerio de Salud de Perú. Alerta epidemiológica. Incremento del riesgo de brotes de dengue de gran magnitud y severidad por la circulación del genotipo asiático/americano del serotipo 2 del virus dengue en el Perú [Internet]. Lima: MINSA; 2011 [citado el 14 de febrero 2015] Disponible en: http:// www.inr.gob.pe/transparencia/ Epidemiolog\%C3\%ADa/alertas\%20 epidemiologicas/ALERTA+EPI DEMIOL\%C3\%93 GICA + N\% C 2\%BA+09-2011-DENGUE 9+NOV+2011.pdf

28. Ministerio de Salud de Perú. Situación de la gestión de las enfermedades metaxénicas [Internet]. Lima: MINSA; 2012 [citado el 14 de febrero 2015] Disponible en: ftp://ftp2.minsa.gob.pe/ donwload/esn/metaxenicas/situacion_ gestion_Enf_Metaxenicas.pdf

29. Ministerio de Salud de Perú. Experiencias exitosas en la prevención y control del dengue en el Perú [Internet].
Lima: ORAS-CONHU; 2012 [citado el 12 de febrero 2015]. Disponible en: http://www.orasconhu.org/sites/ default/files/files/ppt\%20PERU\%20 Reunion $\% 20$ dengue $\% 20$ gye $\% 20$ may2012\%20dR\%20qUINTANA.pdf

30. Decreto Supremo que aprueba el Plan Nacional Multisectorial e Intergubernamental de Prevención y Control de Dengue en Perú. El Peruano. Decreto Supremo N. ${ }^{\circ} 007-$ 2013-SA [18 de junio 2013].

31. Ministerio de Salud de Perú. Guía de práctica clínica para la atención de casos de dengue en el Perú. Lima: MINSA; 2011 [citado el 15 de agosto 2015] Disponible en: http://bvs.minsa.gob. pe/local/MINSA/1377.pdf

32. Ministerio de Salud de Perú. Norma técnica de salud para la implementación de la vigilancia y control de Aedes aegypti, vector del dengue en el territorio nacional. Lima: MINSA; 2010 [citado el 15 de agosto 2015] Disponible en: http://bvs.minsa.gob. pe/local/MINSA/1372.pdf

33. Ministerio de Salud de Perú. Plan integrado de vigilancia, prevención y control del dengue 2011 de la Dirección de Salud V Lima ciudad. Lima: MINSA; 2011. [citado el 15 de agosto 2015] Disponible en: http://bvs. minsa.gob.pe/local/MINSA/1654.pdf

34. Dirección General de Epidemiología, Ministerio de Salud de Perú. Situación de las enfermedades bajo vigilancia epidemiológica en el Perú, al 3 de enero de 2015. Bol Epidemiol (Lima) [Internet]. 2014;23(53):102257 [citado el 27 de febrero 2015]. Disponible en: http://www.dge. gob.pe/portal/docs/vigilancia/ boletines/2014/53.pdf

35. Lozano JM. De patos, gansos y cisnes. Revisiones narrativas, revisiones sistemáticas y metaanálisis de la literatura. Acta Med Colomb. 2005;30(1):1-3.

Correspondencia: Raimundo Castro Orozco Dirección: Universidad de Cartagena, Cartagena de Indias, Colombia Teléfono: (575) 6568031

Correo electrónico: raimundo_castro_ orozco@hotmail.com 